\title{
Reticence and the upper limit
}

\begin{abstract}
People who have nothing much to say, but that they have failed to substantiate another other fellow's claims, seem to follow those with great boasts to make by concealing their messages.
\end{abstract}

This seems to be the era of the phenomena which are not, of the startling observations which seem for a time to stand the world on its head, but which are not afterwards confirmed. By now, almost everybody is familiar with the research reports whose abstracts finish with some such sentence as "... we have therefore established an upper limit of $2 \times 10^{7}$ " with just the suspicion of being the announcement of a discovery in its own right and not the end of some other poor fellow's hopes.

For those with a taste for the macabre, a recent ( 2 May) issue of Physical Review Letters seems to have an unusually rich harvest of upper-limit articles. There is, for example, another contribution to the young but now-rich literature of the fifth force (V. L. Fitch, M. V. Isaila and M. A. Palmer, all Princeton physicists).

The case, it will be recalled, is that of the announcement by E. Fischbach et al. at the beginning of 1986 that inconsistencies in the original data of Eötvös on the gravitational attraction between objects of different materials could be neatly explained if there is an "extra" force of intermediate range dependent on the baryon number (how many nucleons) of the interacting materials.

It is admirable in the true Latin sense, but not really surprising, that the rich crop of experimental articles since to have appeared are nicely contradictory. At a guess, perhaps one article in four puts flesh on the Fischbach conjecture, which was experimental only in the sense of being a re-examination of data collected more than half a century earlier. The others are "upper-limit" articles which, however objective, tend to reinforce the impression that the fifth force may have made only a brief appearence on the scene (not that that will deter people from trying to measure the force, nor should it).

Fitch et al. have shown what remarkable steps people will take to secure data in the field. They have repeated the Eötvös measurements on the north-sloping face of Mt Maurice at Yellowstone, Montana. A hint of the difficulty is provided by the tangent of the slope of the mountainside to the South (and above), quoted as 0.5.

Yet, fair play, there is not a hint of disappointment at the obvious scatter of the crude measurements, or in the estimates of the parameters defining the supposed extra force which are all accompanied by errors larger than themselves.
Laconically, the abstract says that "...we find the product of strength $a_{0}$ and range $\tau$ to be $-0.04 \pm 0.07 \mathrm{~m} . . . "$. In the text, the authors say "...these results agree with those in Refs. 8, 9 and 11 , and are in disagreement with those in Ref.12..." without telling their readers that "Ref.12" is P. Thieberger's affirmative measurement of the fifth force on the Palisades cliffside opposite Manhattan. Those wishing to read upper-limit articles intelligently must first learn the sign-language of the calculated understatement.

Some of the same applies to a more spectacular upper-limit article in the same issue by a group (B.L.Dingus et al. 60, 1785 ; 1988) scattered between the University of Maryland, Los Alamos, the University of New Mexico and the University of California, Irvine, which has been looking for evidence that Cygnus X-3 (the Xray star with a binary orbital period of 4.8 hours) is a powerful source of energetic $\gamma$-rays, with energy exceeding $10^{16} \mathrm{eV}$. Plainly it is a matter of some importance that the issue should be pinned down.

The need persists for sign-language in reading this article as well, but one is helped along a little more. The inference that the periodically emitted energetic particles must be $\gamma$-rays derives from a simple estimate that only electrically neutral particles could successfully navigate intergalactic space without dispersion that would entirely obscure the 4.8 hour periodicity observed in the X-ray flux, but there have been (since 1983) reports of $\mu$ mesons as well. So Dingus et al. have built a powerful cosmic-ray shower detector at Los Alamos, giving themselves the luxury of detecting accompanying muons of sufficient energy (above $2 \mathrm{GeV}$ ) individually.

Sadly, the outcome of all this sophistication is that the periodicity of Cygnus X-3 as a source of $50 \mathrm{TeV} \gamma$-rays is not confirmed - but you would not guess that from the abstract. The skeleton of the main sentence is "Upper limits for ... the flux of air-showers from ... Cygnus X-3 ...are found to be $1 \times 10^{-13} \mathrm{~cm}^{-2} \mathrm{~s}^{-1}$ for all events, and ... for all events with no observed muons...". If one asks how these fluxes compare with those which have stimulated these measurements, one must presumably go burrowing in the references.

But this paper does have two saving graces. First, buried away towards the end of the text is an explicit sentence reading "data from the entire run show no statistically significant signal ... from the direc- tion of Cygnus X-3." Second, there follows simple declaration that there was a period of 45 days (out of a total of 264) when there were 27 extensive showers that might have been correlated with the phase of the distant X-ray star even though "... the statistical evidence is not compelling".

That, for what it is worth, is not the end of it. The same issue of the same journal has no fewer than two calculations of upper (or lower) bounds for the properties of the particle called the axion derived from the observation of neutrinos from last year's supernova, 1987A. (One of them, without as much inconsistency as there might seem to be, derives both an upper and a lower bound for the axion mass, but puts the former below the latter: the argument is that calculations of what will happen to axions depend crucially on whether they are massive enough to be trapped in the supernova gravitationally, or whether they move without constraint.)

There is a temptation to make fun of such logic-chopping, but there is a practical problem to be solved by those who engage in it, or on their behalf. First, as with the fifth force, it is in no sense surprising, but the opposite, that the announcement of a new phenomenon should be followed by a rash of confirmatory and contradictory results. By definition, the first measurement will have been at the edge of what is technically possible; those that follow, at least at first, are likely to be less expert and therefore less sensitive. That is why those breaking new ground should not be downcast that most of those who flatter them by repeating their measurement end up in disagreement.

Second, the credentials of those who repeat others people's measurements should be examined, not so much for evidence of cynicism but the opposite. There may be occasions when a person sets out to repeat another's experiment in the expectation that he will find an error, but more often it must be the case that the persons who spent all those months building equipment must have believed they would find something, not find something else wrong. Third, there is a matter of prose style. People who may be prevented, by modesty or some such consideration, from saying in plain language what they have found out, might be expected to name names when their conclusion fails to confirm what somebody else has claimed. But the convention seems to go the other way, which is a pity.

John Maddox 